\title{
EVOLUCIÓN Y DIFERENCIAS REGIONALES DE LA MORTALIDAD POR SUICIDIOS EN EL PERÚ, 2004-2013
}

\author{
Akram Hernández-Vásquez ${ }^{1, a, f}$, Diego Azañedo ${ }^{2, b}$, Juan Rubilar-González $z^{3, c}$, Bertha Huarez ${ }^{4, d}$, Leandro Grendas ${ }^{1, e, g}$

\begin{abstract}
RESUMEN
El objetivo del estudio fue estimar y analizar la evolución de las tasas de mortalidad por suicidios en el Perú entre los años 2004 y 2013. Se analizaron los registros nacionales de defunciones del Ministerio de Salud del Perú, calculando las tasas regionales de mortalidad por suicidios estandarizadas por edad. Asimismo, se proyectaron geoespacialmente las tasas agrupadas por quinquenios. Se identificaron 3162 casos de suicidios $(67,2 \%$ hombres), el grupo etario con mayor incidencia fue el de 20 a 29 años $(28,7 \%)$ y el $49,2 \%$ ocurrieron por envenenamiento. La tasa de suicidios pasó de 0,46 (IC 95\%: 0,38-0,55) a 1,13 (IC 95\%: 1,01-1,25) por 100000 habitantes en el 2004 y 2013, respectivamente. Las mayores tasas se identificaron en Pasco, Junín, Tacna, Moquegua y Huánuco. El problema de los suicidios en Perú requiere de un abordaje integral que implica no solo identificar zonas con mayor riesgo, sino también estudiar sus factores asociados que podrían explicar la variabilidad regional observada.
\end{abstract}

Palabras clave: Suicidio; Mortalidad; Sistemas de Información Geográfica; Perú (fuente: DeCS BIREME).

\section{EVOLUTION AND REGIONAL DIFFERENCES IN MORTALITY DUE TO SUICIDE IN PERU, 2004-2013}

\begin{abstract}
The aim of this study was to estimate and analyze the evolution of mortality rates due to suicide in Peru between 2004 and 2013. National death records from the Peruvian Ministry of Health were analyzed, calculating the regional mortality rates due to suicide standardized by age. Similarly, rates grouped in 5-year periods were geospatially projected. There were 3,162 cases of suicide $(67.2 \%$ men); the age range with the highest incidence was 20 to 29 years $(28.7 \%)$ and $49.2 \%$ were due to poisoning. Suicide rates increased from $0.46(95 \%$ confidence interval $[\mathrm{Cl}]=0.38-0.55)$ to $1.13(95 \%$ $\mathrm{Cl}=1.01-1.25$ ) per 100,000 people from 2004 to 2013, respectively. The highest rates of suicide were identified in Pasco, Junín, Tacna, Moquegua, and Huánuco. The suicide issue in Peru requires a comprehensive approach that entails not just identifying the areas with the highest risk, but also studying its associated factors that may explain the regional variability observed.
\end{abstract}

Key words: Suicide; Mortality; Geographic Information Systems; Peru (source: MeSH NLM).

\section{INTRODUCCIÓN}

El suicidio es un fenómeno complejo e independiente que, en los últimos 45 años, ha presentado un incremento en un $60 \%$ a nivel mundial ${ }^{(1)}$. En el 2008 se registraron 782000 suicidios, cifra que aumentó a 804000 para el año 2012, llegando a estimarse una tasa de suicidio de 11,4 por cada 100000 habitantes $(15,0$ entre hombres y 8,0 entre mujeres), y aproximadamente 20 intentos de suicidio por cada suicidio consumado ${ }^{(1,2)}$. Este incremento posicionó a los suicidios como la segunda causa de muerte entre personas de 15 a 29 años a nivel mundial (1). Aun siendo los suicidios prevenibles en la mayoría de los casos, es una de las principales causas de años de vida perdidos por enfermedad (AVP), y en los EE.UU. es responsable de un costo de alrededor de 44,6 billones de dólares anuales entre gastos médicos y pérdidas laborales ${ }^{(3,4)}$.

Aproximadamente, el $90 \%$ de suicidastienenantecedentes de desórdenes mentales, siendo los más comunes la depresión y el abuso de sustancias ${ }^{(1)}$. De igual manera, desde la tipificación de las causas estructurales del suicidio en la obra de Durkheim hasta estudios recientes,

\footnotetext{
Universidad de Buenos Aires. Buenos Aires, Argentina.

Instituto de Investigación, Universidad Católica los Ángeles de Chimbote. Chimbote, Perú.

Universidad Mayor. Temuco, Chile.

4 Oficina General de Información y Sistemas, Instituto Nacional de Salud. Lima, Perú.

a Médico cirujano; ${ }^{\mathrm{b}}$ cirujano dentista; ${ }^{\mathrm{c}}$ sociólogo; ${ }^{\mathrm{d}}$ licenciada en Educación; ${ }^{\mathrm{e}}$ médico; ${ }^{\mathrm{f}}$ magíster en Gestión y Políticas Públicas; ${ }^{\mathrm{g}}$ especialista en Psiquiatría. Recibido: 25/05/2016 Aprobado: 23/11/2016
} 
se han identificado potenciales factores de riesgo de suicidio como el aislamiento social, intentos previos, imitación, desempleo, enfermedad física y conflictos familiares ${ }^{(5,6)}$ que son muy comunes y repercuten con mayores consecuencias en los países en desarrollo ${ }^{(7,8)}$. Asimismo, se han reportado en América Latina factores de riesgo asociados a intento de suicidio tales como el sexo femenino, tentativa previa, trastornos mentales, intoxicación alcohólica previa a la tentativa, estructura familiar disfuncional y bajo apoyo social; así también, factores de riesgo asociados a suicidio consumado tales como ser de sexo masculino y presentar un trastorno depresivo mayor ${ }^{(9)}$.

En el Perú, se estima que el $25 \%$ de la población sufre de depresión, y que de estos un $15 \%$ es considerado como grupo de riesgo de suicidio; además, los motivos de la decisión suicida responden, en su mayoría, a conflictos de pareja y familiares ${ }^{(10-12)}$. La tasa de suicidio ha aumentado de 0,9 en el año 2000 hasta 1,01 en el año 2009 por cada 100000 habitantes ${ }^{(13,14)}$, mostrando un crecimiento que podría seguir aumentando y que genera la necesidad de estudiar en profundidad sus factores asociados.

Por otro lado, se debe tener en cuenta que la ocurrencia de suicidios es variable geográficamente entre y dentro de los países, posiblemente por las disparidades en las condiciones socioeconómicas, prevalencia de comorbilidades físicas y mentales y acceso a atención especializada ${ }^{(15)}$. En el Perú aún no existen estudios sobre el perfil epidemiológico del suicidio en las regiones, lo cual limita la disponibilidad de evidencias que permitan una efectiva instauración de medidas preventivas que reduzcan su incidencia. Siendo así, es necesario analizar este problema tanto a nivel nacional como local, de modo que se puedan detectar diferencias y tendencias que permitan inferir posibles factores de riesgo donde sea necesario enfocar la atención ${ }^{(16,17)}$.

Este artículo tiene por objetivo estimar y analizar la evolución de las tasas de mortalidad por suicidios en el Perú y sus regiones entre los años 2004-2013, que permita aportar evidencias para una mejor toma de decisiones en el ámbito de la salud pública.

\section{EL ESTUDIO}

Se realizó un análisis secundario de los registros de defunciones proporcionados por la Oficina General de Estadística e Informática del Ministerio de Salud del Perú (MINSA) de los últimos diez años disponibles (2004-2013). Los datos incluyeron todas aquellas defunciones registradas a nivel nacional y según región de ocurrencia de cada suicidio bajo los códigos X60-X84 de la décima versión de la Clasificación Internacional de Enfermedades (CIE-10). Los datos poblacionales regionales para cada año de estudio fueron obtenidos de la Series Nacionales del Instituto Nacional de Estadística e Informática del Perú (http://webinei.inei. gob.pe:8080/sirtod-series/).

Se utilizó el software estadístico Stata 14.0 (StataCorp LP, College Station, TX, USA), para mostrar frecuencias, proporciones y/o medias para las categorías incluidas; así como también, para obtener tasas regionales de mortalidad general estandarizadas por edad para cada una de las regiones del Perú, y una tasa agrupada por quinquenios (2004-2008 y 2009-2013) expresadas por cada 100000 habitantes. La estandarización fue realizada por el método directo según el estándar de población de la Organización Mundial de la Salud (OMS) ${ }^{(18)}$. Asimismo, la prueba t de Student fue aplicada para establecer diferencias de las edades promedio de suicidios entre hombres y mujeres. Las tasas obtenidas fueron incluidas en una hoja de cálculo de Microsoft Excel ${ }^{\circledR} 2013$ (Microsoft, Redmond, WA) para analizar la evolución temporal de las tasas de suicidio y elaboración de figuras.

Finalmente, las tasas ajustadas de los quinquenios para cada una de las regiones fueron proyectadas a un mapa regional del Perú haciendo uso del software ArcGIS versión 10.4 (ESRI Inc., Redlands, CA, USA) para una mejor visualización e identificación de las regiones con mayores y menores tasas, e identificar posibles cambios entre quinquenios.

El estudio no requirió la aprobación de un comité de ética por tratarse de un estudio ecológico que utiliza datos secundarios agrupados que no permite identificar a los sujetos cuyas defunciones fueron registradas.

\section{HALLAZGOS}

Un total de 3162 suicidios fueron registrados en la base de datos nacional de defunciones del MINSA en el periodo 2004-2013. Del total de eventos reportados, un $67,2 \%$ fueron hombres; el grupo etario de 20 a 29 años fue el grupo de mayor incidencia con $28,7 \%$ del total de casos, y el $49,2 \%$ de eventos ocurrió por envenenamiento. El promedio de edad de los suicidas fue de 34,5 años. La media de edad de suicidio en hombres (36,7 años) fue más alta comparada con la de las mujeres $(30,1$ años) $(p=0,001)$. La región de la sierra mostró la mayor incidencia $(71,0 \%)$ seguida de la costa $(22,4 \%)$ y la selva $(6,6 \%)$. No hubo variación en la proporción de ocurrencia de suicidios entre ocupados: $43,2 \%$ vs. desocupados $43,4 \%$, y el estado civil soltero tuvo el mayor número de casos $(42,1 \%)$ (Ver Tabla 1$)$.

La Figura 1 muestra cómo las tasas de suicidios han presentado variabilidad y una tendencia creciente a través de los años. La tasa de suicidios ajustada por 
Tabla 1. Características generales de suicidios ocurridos en el Perú durante el periodo 2004-2013 ( $\mathrm{N}=3162)$

\begin{tabular}{|c|c|c|}
\hline \multirow[t]{2}{*}{ Características } & \multicolumn{2}{|c|}{$\begin{array}{l}\text { Número y proporción de } \\
\text { suicidios }\end{array}$} \\
\hline & $\mathbf{N}$ & $\%$ \\
\hline \multicolumn{3}{|l|}{ Sexo } \\
\hline Masculino & 2124 & 67,2 \\
\hline Femenino & 1038 & 32,8 \\
\hline \multicolumn{3}{|l|}{ Grupos etarios (años) } \\
\hline $10-14$ & 151 & 4,8 \\
\hline $15-19$ & 549 & 17,4 \\
\hline $20-29$ & 907 & 28,7 \\
\hline $30-44$ & 725 & 22,9 \\
\hline $45-64$ & 571 & 18,1 \\
\hline 65 a más & 259 & 8,2 \\
\hline \multicolumn{3}{|l|}{ Tipo de suicidio } \\
\hline Envenenamiento & 1557 & 49,2 \\
\hline Plaguicidas & 597 & 18,9 \\
\hline Otros $^{*}$ & 960 & 30,3 \\
\hline Ahorcamiento & 573 & 18,1 \\
\hline Arma de fuego & 83 & 2,6 \\
\hline Otros & 37 & 1,2 \\
\hline No especificado & 912 & 28,8 \\
\hline \multicolumn{3}{|l|}{ Región natural } \\
\hline Costa & 708 & 22,4 \\
\hline Sierra & 2246 & 71,0 \\
\hline Selva & 208 & 6,6 \\
\hline \multicolumn{3}{|l|}{ Ocupación } \\
\hline Ocupado & 1367 & 43,2 \\
\hline Desocupado & 1371 & 43,4 \\
\hline Ignorado & 424 & 13,4 \\
\hline \multicolumn{3}{|l|}{ Estado civil } \\
\hline Soltero & 1331 & 42,1 \\
\hline Conviviente & 778 & 24,6 \\
\hline Casado & 616 & 19,5 \\
\hline Divorciado & 169 & 5,3 \\
\hline Viudo & 158 & 5,0 \\
\hline Ignorado & 110 & 3,5 \\
\hline
\end{tabular}

* Incluye grupos de códigos CIE 10: X60, X61, X62, X63, X64, X65, X66, X67 y X69

edad pasó de 0,46 (IC 95\%: 0,38-0,55) por 100000 habitantes en 2004 a 1,13 (IC 95\%: 1,01-1,25) por 100000 habitantes en el 2013. Asimismo, la tasa de suicidios ajustada por edad fue más alta para hombres comparada con mujeres durante el periodo de estudio.

La Figura 2A muestra la proyección temporal de porcentaje relativo de suicidios en función del sexo. Se puede apreciar que los hombres representan la mayor proporción de casos frente a las mujeres. Adicionalmente, puede observarse que la proporción relativa de mujeres fue más alta en 2005 que en otros años, con un marcado descenso hacia el 2009. En la Figura 2B se representan los diferentes tipos de suicidio. Dentro de los tipos específicos de suicidios, el envenenamiento es el más frecuente durante el periodo

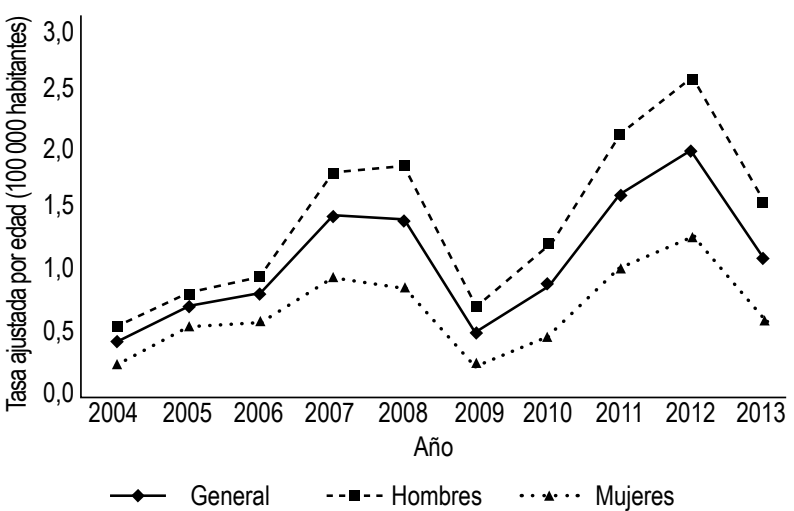

Figura 1. Evolución de las tasas de mortalidad por suicidios 2004-2013 en el Perú

de estudio, seguido por el ahorcamiento y las armas de fuego. Asimismo, el envenenamiento tuvo la proyección más constante hasta el año 2009, luego del cual, el ahorcamiento empezó a tener mayor frecuencia.

Según regiones, las mayores tasas de suicidios ajustadas por edad se identificaron en Pasco el año 2013 con 8,85 suicidios por 100000 habitantes seguidos
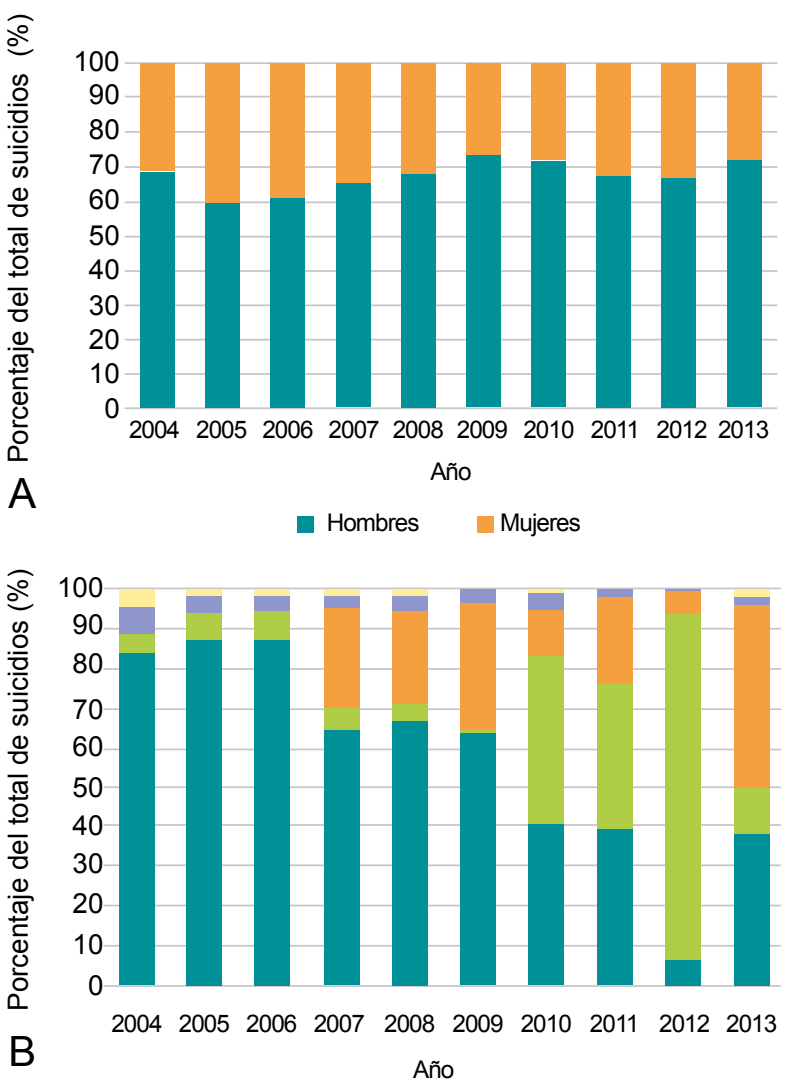

Envenenamiento $\quad$ No especificado $\quad$ Ahorcamiento
Arma de fuego Otros

Figura 2. A Proporción de suicidios según sexo y año durante el periodo 2004-2013. B Proporción de suicidios según tipo y año durante el periodo 2004-2013 
por Junín $(8,32)$ en el 2011 y Tacna junto con Moquegua $(8,28)$ en el 2007 ; mientras algunas otras presentaron tasas muy bajas o nulas en algunos años como Loreto, Madre de Dios, Tumbes y Lambayeque (Anexo 1).

Las tasas de suicidios ajustadas por edad del periodo 2004-2008 y 2009-2013 según regiones se pueden

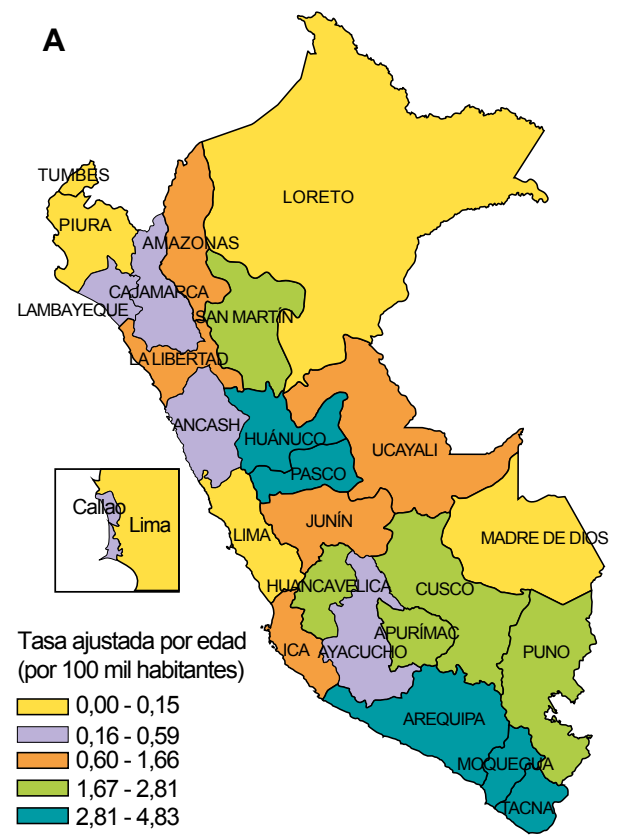

observar en la Figura 3, con mayores tasas que fluctúan entre el rango de 2,81 a 4,83 para el periodo 2004-2008; y de 2,31 a 6,00 para 2009-2013. Cabe precisar que, en ambos casos, las regiones con mayores tasas por cada 100000 habitantes fueron Arequipa, Pasco, Tacna y Huánuco.

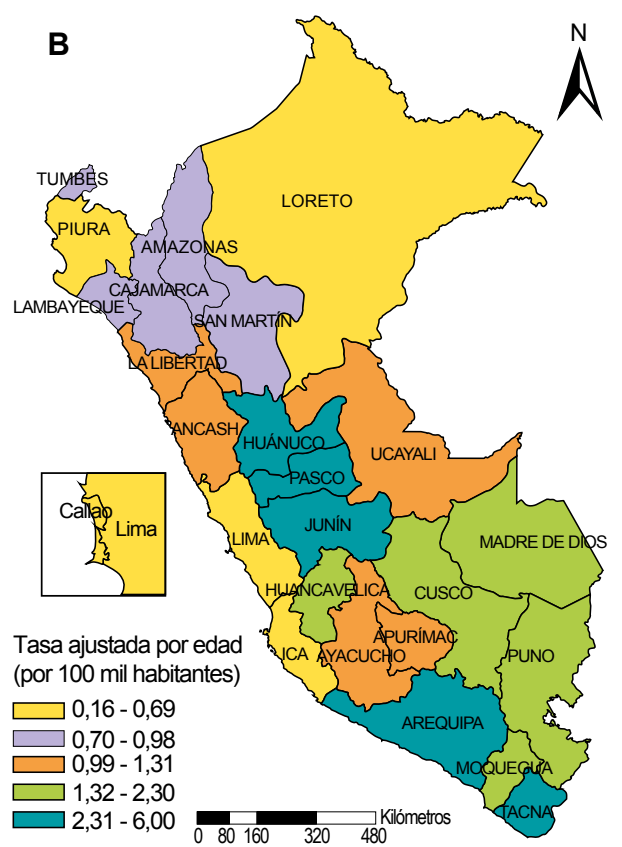

Figura 3. A Tasa de suicidios ajustada por edad para el periodo 2004-2008 según regiones. B Tasa de suicidios ajustada por edad para el periodo 2009-2013 según regiones.

\section{DISCUSIÓN}

Se identificó una variabilidad en la evolución de las tasas de suicidios en el Perú, y al igual que otros países de la región, existe un incremento del número de casos entre el periodo 2004-2013; no obstante, aún las cifras están muy por debajo de países como Chile o Uruguay ${ }^{(14)}$. Además, las tasas se muestran elevadas en las regiones de Arequipa, Pasco, Junín y Huánuco. Estas regiones se caracterizan por ser zonas andinas que han tenido diversos conflictos sociales en el pasado; hay déficit de profesionales de la salud mental; tienen altas prevalencias de abuso/dependencia de alcohol, seguido de depresión y trastorno de ansiedad generalizada; que podrían tener cierta influencia en las tasas halladas ${ }^{(19,20)}$.

En concordancia con los datos a nivel mundial, se observó un patrón claro de predominio del sexo masculino entre aquellos que consuman un suicidio. Las posibles explicaciones de este fenómeno apuntan a que los hombres usan métodos más letales para suicidarse como el uso de armas de fuego o el ahorcamiento; mientras las mujeres optan como plan suicida la ingesta de drogas o tóxicos que, en algunas ocasiones, pueden recibir atención oportuna con mayores posibilidades de sobrevivir; asimismo, las mujeres podrían tener una mejor predisposición para superar ciertos eventos de la vida, que en algunos casos pueden ser desencadenante de suicidios ${ }^{(15,21,22)}$.

Al igual que otros países de la región y el mundo, el mayor número de defunciones por suicidio se observó en el grupo de 20 a 29 años, coincidiendo con los datos mundiales donde figura el suicidio dentro de las principales causas de muerte en adultos jóvenes ${ }^{(1,15)}$. Teniendo en cuenta que la edad avanzada es un predictor de intento de suicidio fatal (23), y que tanto en Perú como en otros países de la región se registra un descenso en las tasas de suicidio a medida que aumenta la edad, se requiere de estudios prospectivos que avalúen la influencia de la edad en el comportamiento suicida.

Cabe resaltar que en este estudio se identificó al envenenamiento como principal tipo de suicidio, mientras en otros países de la región como Chile o 
Brasil, el método más utilizado fue el ahorcamiento o las armas de fuego ${ }^{(24,25)}$. Este hecho podría estar ocurriendo debido a la fácil accesibilidad que se tiene en el Perú a pesticidas o venenos para roedores, cuya ingesta es fatal en humanos. Esto debería ser tomado en cuenta para la realización de políticas de salud, ya que la restricción del acceso a los métodos empleados para el plan suicida es clave para la prevención del suicidio (1). A pesar de estos hallazgos generales, en el año 2013 se registró una disminución de los casos de envenenamiento, en términos relativos, a casi la mitad con respecto a los años 2004, 2005 y 2006, siendo mayor el número de casos por ahorcamiento, lo que podría indicar una variación en la tendencia de los tipos de suicidio.

La principal limitación de este tipo de estudios es el subregistro de los eventos de mortalidad, considerando el elevado número de eventos sin especificar el tipo de suicidio según CIE-10 y los notorios descensos en la mortalidad de los años 2009 y 2013; situación que podría indicar la existencia de un mayor número de suicidios que no son registrados. No obstante, estos descensos se corresponden con las cifras reportadas por la OMS (http://apps.who.int/healthinfo/statistics/mortality/ whodpms/), las cuales presentan tasas con oscilaciones desde el año 1983; a pesar de esto, consideramos que nuestros resultados contribuyen a mostrar la realidad de los suicidios a nivel de las regiones, haciendo uso de cifras oficiales. Cabe precisar que si bien el CIE-10 puede no ofrecer un detalle suficiente sobre los métodos suicidas, consideramos que este tipo de precisiones podrían ser incluidas en un artículo con mayor amplitud o en uno de revisión de la nueva versión del CIE (Versión 11) que está en fase piloto en algunos países. Por estos motivos es importante mencionar la necesidad de contar con un buen sistema de información y vigilancia de este problema de salud pública de modo que permita identificar grupos de mayor riesgo, evaluar patrones cambiantes debido a características o contextos asociados con un mayor riesgo y la evaluación de la efectividad de las actuales medidas de prevención (1).

Los pocos estudios realizados en el Perú sobre suicidios han evaluado la relación existente entre factores de riesgo para grupos etarios o subpoblaciones, sin tener en cuenta la diversidad territorial o socioeconómica del país ${ }^{(11-12) ; ~ p o r ~ l o ~}$ que considerarlas permitirá un mejor abordaje y disposición de los recursos que se dispongan para contrarrestarlos. Ante ello, nuestros hallazgos representan un avance para el análisis de la distribución de las tasas de suicidio en cada región del país principalmente porque representa un problema complejo que tiene mayor impacto en poblaciones vulnerables.

En conclusión, en el presente estudio se encontró una evolución incremental de la mortalidad por suicidios en el Perú; así como, grandes diferencias entre las regiones. Lo anterior pone en evidencia la necesidad de enfrentar este problema a corto plazo y nos plantea el reto de un abordaje integral mediante un conjunto de medidas que requieren no solo identificar zonas con mayor riesgo de ocurrencia, sino también estudiar aquellos factores relacionados a ella, especialmente los problemas de salud mental y los factores locales que influyen en la conducta suicida, que podrían explicar la variabilidad observada en las distintas regiones.

Fuentes de financiamiento: autofinanciado.

Contribuciones de autoría: AHV tuvo la idea de investigación. AHV y DA diseñaron el estudio y recopilaron los datos. AHV realizó el análisis estadístico y distribución espacial de los datos. AHV, DA, JRG, BH y LG analizaron e interpretaron los datos, redactaron el manuscrito y aprobaron la versión final.

Conflictos de interés: los autores declaran no tener conflictos de interés.

\section{REFERENCIAS BIBLIOGRÁFICAS}

1. Organización Mundial de la Salud. Prevención del suicidio: un imperativo global [Internet]. Washington, DC: OPS; 2014 [Citado el 7 de abril de 2016]. Disponible en: http://apps.who.int/iris/bitstre am/10665/136083/1/9789275318508_ spa.pdf.

2. Värnik P. Suicide in the World. Int J Environ Res Public Health. 2012;9(3):760-71. doi: 10.3390/ ijerph9030760

3. Center for Disease Control and Prevention. Suicide: Consequences [Internet]. Atlanta: CDC; 2015
[Citado el 13 de abril de 2016]. Disponible en: http://www.cdc. gov/violenceprevention/suicide/ consequences.html.

4. Vigo D, Thornicroft G, Atun R. Estimating the true global burden of mental illness. Lancet Psychiatry. 2016;3(2):171-8. doi: 10.1016/S22150366(15)00505-2

5. Franklin JC, Ribeiro JD, Fox KR, Bentley KH, Kleiman EM, Huang X, et al. Risk Factors for Suicidal Thoughts and Behaviors: A Meta-Analysis of 50 Years of Research. Psychol Bull. 2016;e46 Epub 2016 Nov 14.
6. Chang BP, Franklin JC, Ribeiro JD, Fox KR, Bentley KH, Kleiman EM, et al. Biological risk factors for suicidal behaviors: a meta-analysis. Transl Psychiatry. 2016;6(9):e887. doi: 10.1038/tp.2016.165

7. Khan MM. Suicide prevention and developing countries. J R Soc Med. 2005;98(10):459-63. doi: 10.1258/ jrsm.98.10.459

8. Vijayakumar L, John S, Pirkis J, Whiteford H. Suicide in developing countries (2): risk factors. Crisis. 2005;26(3):112-9. doi: 10.1027/02275910.26 .3 .112 
9. Teti GL, Rebok F, Rojas SM, Grendas L, Daray FM. Systematic review of risk factors for suicide and suicide attempt among psychiatric patients in Latin America and Caribbean. Rev Panam Salud Publica. 2014;36(2):124-33.

10. Instituto Nacional de Salud del Niño. Boletín de la Unidad de Investigación Epidemiológica y Análisis Situacional de Servicios de Salud [Internet]. Lima: Oficina de Epidemiología, INSN; 2014 [Citado el 11 de abril de 2016]. Disponible en: http:// www.insn.gob.pe/sites/default/files/ Boletin\%20Sala\%20Situacional\%20 DICIEMBRE\%202014.pdf.

11. Coronado-Molina O. Características clínico-epidemiológicas y abordaje terapéutico de pacientes que acuden por intento suicida al Hospital General San José del Callao, Perú. Rev Neuropsiquiatr. 2014;77(4):242-9.

12. Castro P, Orbegoso O, Rosales P. Caracterización del paciente con intento de suicidio en un hospital general de Lima, 1995-2004. Rev Peru Med Exp Salud Publica. 2006;23(4):293-6.

13. World Health Organization. Suicide rates Perú [Internet]. Geneva Switzerland: WHO [Citado el 8 de abril de 2016]. Disponible en: http://www.who.int/ mental_health/media/peru.pdf.
14. Organización Panamericana de la Salud. Mortalidad por suicidio en las Américas: Informe regional [Internet]. Washington, DC: OPS; 2014 [Citado el 8 de abril de 2016]. Disponible en: http://www.paho.org/hq/index. php?option $=$ com_docman $\&$ task $=$ doc download\&Itemid=\&gid=27709\&lang=es.

15. Pan American Health Organization. Prevention of suicidal behavior [Internet]. Washington, DC: PAHO; 2016 [Citado el 15 de noviembre de 2016]. Disponible en: http://iris.paho.org/xmlui/bitstream/han dle/123456789/31166/9789275119198eng.pdf? sequence= 1 \&isAllowed=y.

16. Phillips $M$. Suicide prevention in developing countries: where should we start? World Psychiatry. 2004;3(3):156-7.

17. Jalles JT, Andresen MA. The social and economic determinants of suicide in Canadian provinces. Health Econ Rev. 2015;5:1. doi: 10.1186/s13561-015-0041-y

18. Ahmad O, Boschi-Pinto C, Lopez A, Murray C, Lozano R, Inoue M. Age standardization of rates: a new WHO standard. [Internet]. Geneva:WHO;2000 [Citado el 8 de abril de 2016]. Disponible en: http://www.who.int/ healthinfo/paper31.pdf?ua=1.

19. Soberón R. Los usuarios de drogas en el Perú, políticas, derechos y problemas [Internet]. [Citado el 24 de mayo de 2016]. Disponible en: http://www. drogasyderecho.org/publicaciones/pubper/peru-usuarios.pdf.

20. Cueva G. Violencia y adicciones: problemas de salud pública. Rev Peru
Med Exp Salud Publica. 2012;29(1):99103.

21. Mościcki EK. Gender differences in completed and attempted suicides. Ann Epidemiol. 1994;4(2):152-8.

22. Rich CL, Ricketts JE, Fowler RC, Young D. Some differences between men and women who commit suicide. Am J Psychiatry. 1988;145(6):718-22.

23. Beghi M, Rosenbaum JF, Cerri C, Cornaggia CM. Risk factors for fatal and nonfatal repetition of suicide attempts: a literature review. Neuropsychiatric Dis Treat. 2013;9:1725-36. doi: 10.2147/ NDT.S40213

24. Bustamante F, Ramirez V, Urquidi C, Bustos V, Yaseen Z, Galynker I. Trends and Most Frequent Methods of Suicide in Chile Between 2001 and 2010. Crisis. 2016;37(1):21-30. doi: 10.1027/02275910/a000357

25. Palacio C, Garcia J, Diago J, Zapata C, Lopez G, Ortiz J, et al. Identification of suicide risk factors in Medellin, Colombia: a case-control study of psychological autopsy in a developing country. Arch Suicide Res. 2007;11(3):297-308. doi: $10.1080 / 13811110600894223$

Correspondencia: Akram Abdul
Hernández Vásquez
Dirección: Calle Gavilán 3856, Ciudad
Autónomade Buenos Aires, Argentina
Teléfono:(0054)91122981572
Correo electrónico: akram.hernandez.v@upch.pe

\section{Nuestros artículos se encuentran indizados en:}

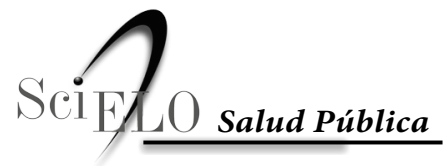


Anexo 1. Tasa de suicidios ajustada por edad según regiones del Perú, 2004-2013

\begin{tabular}{|c|c|c|c|c|c|c|c|c|c|c|c|}
\hline \multirow{2}{*}{ Región } & \multirow{2}{*}{$\begin{array}{c}\text { Población } \\
2013\end{array}$} & \multicolumn{10}{|c|}{ Tasa ajustada por edad (por 100000 habitantes) } \\
\hline & & 2004 & 2005 & 2006 & 2007 & 2008 & 2009 & 2010 & 2011 & 2012 & 2013 \\
\hline Amazonas & 419404 & 2,15 & 1,72 & 0,34 & 0,00 & 0,00 & 0,00 & 0,40 & 0,87 & 1,82 & 0,67 \\
\hline Ancash & 1135962 & 0,27 & 0,12 & 0,36 & 0,07 & 0,68 & 0,79 & 2,40 & 1,32 & 1,64 & 0,44 \\
\hline Apurímac & 454324 & 0,00 & 1,52 & 1,05 & 4,08 & 1,96 & 0,41 & 0,74 & 1,50 & 2,71 & 1,04 \\
\hline Arequipa & 1259162 & 1,16 & 3,10 & 2,39 & 7,15 & 7,51 & 6,54 & 4,01 & 5,52 & 8,09 & 5,83 \\
\hline Ayacucho & 673609 & 0,79 & 0,41 & 0,12 & 0,58 & 0,61 & 0,23 & 0,69 & 0,62 & 4,33 & 0,24 \\
\hline Cajamarca & 1519764 & 0,26 & 0,35 & 0,51 & 0,89 & 0,89 & 0,30 & 0,67 & 1,27 & 1,58 & 1,04 \\
\hline Callao & 982800 & 0,00 & 0,00 & 0,26 & 0,31 & 0,19 & 0,09 & 0,53 & 0,62 & 1,28 & 0,91 \\
\hline Cusco & 1300609 & 0,10 & 3,42 & 2,86 & 4,29 & 3,23 & 0,52 & 1,22 & 2,45 & 3,25 & 2,72 \\
\hline Huancavelica & 487472 & 1,50 & 2,87 & 2,56 & 0,63 & 1,79 & 0,58 & 1,62 & 0,72 & 3,84 & 0,62 \\
\hline Huánuco & 847714 & 3,27 & 2,02 & 1,99 & 5,81 & 5,30 & 3,27 & 0,76 & 3,30 & 3,50 & 2,72 \\
\hline Ica & 771507 & 0,13 & 0,69 & 0,67 & 0,77 & 0,62 & 0,37 & 1,02 & 0,93 & 0,16 & 0,80 \\
\hline Junín & 1331253 & 0,28 & 0,52 & 1,67 & 1,91 & 3,80 & 0,95 & 2,34 & 8,32 & 5,17 & 1,21 \\
\hline La Libertad & 1814276 & 0,43 & 0,19 & 0,31 & 0,90 & 1,17 & 0,19 & 1,26 & 0,92 & 1,52 & 2,16 \\
\hline Lambayeque & 1239882 & 0,13 & 0,00 & 0,00 & 0,67 & 0,08 & 0,09 & 0,00 & 0,23 & 1,61 & 1,70 \\
\hline Lima & 9540996 & 0,04 & 0,06 & 0,11 & 0,24 & 0,21 & 0,00 & 0,34 & 0,54 & 0,50 & 0,13 \\
\hline Loreto & 1018160 & 0,08 & 0,00 & 0,00 & 0,18 & 0,00 & 0,00 & 0,70 & 0,09 & 0,00 & 0,00 \\
\hline Madre de Dios & 130876 & 0,00 & 0,00 & 0,00 & 0,72 & 0,00 & 0,00 & 0,71 & 2,92 & 7,68 & 0,00 \\
\hline Moquegua & 176736 & 0,55 & 1,13 & 2,21 & 8,28 & 5,10 & 0,00 & 1,28 & 1,04 & 2,57 & 4,27 \\
\hline Pasco & 299807 & 2,76 & 3,93 & 2,62 & 6,04 & 5,07 & 0,00 & 1,28 & 5,43 & 6,77 & 8,85 \\
\hline Piura & 1814622 & 0,06 & 0,15 & 0,38 & 0,00 & 0,05 & 0,00 & 0,19 & 0,79 & 0,56 & 0,55 \\
\hline Puno & 1389684 & 1,08 & 1,87 & 2,96 & 2,69 & 3,91 & 0,00 & 1,31 & 4,23 & 4,65 & 0,62 \\
\hline San Martín & 818061 & 1,49 & 1,51 & 1,81 & 2,33 & 3,01 & 0,00 & 1,52 & 0,62 & 1,68 & 0,39 \\
\hline Tacna & 333276 & 2,72 & 2,38 & 6,67 & 8,28 & 3,76 & 0,00 & 0,76 & 3,98 & 7,55 & 3,58 \\
\hline Tumbes & 231480 & 0,00 & 0,00 & 0,00 & 0,00 & 0,00 & 0,00 & 0,35 & 0,37 & 0,43 & 2,51 \\
\hline Ucayali & 483708 & 1,43 & 0,39 & 0,63 & 3,71 & 1,26 & 0,00 & 0,46 & 3,04 & 1,91 & 0,00 \\
\hline Nacional & 30475144 & 0,46 & 0,74 & 0,84 & 1,47 & 1,44 & 0,52 & 0,92 & 1,64 & 2,02 & 1,13 \\
\hline
\end{tabular}

Nota: Se incluye la población 2013 solo como referencia del tamaño poblacional de la región.

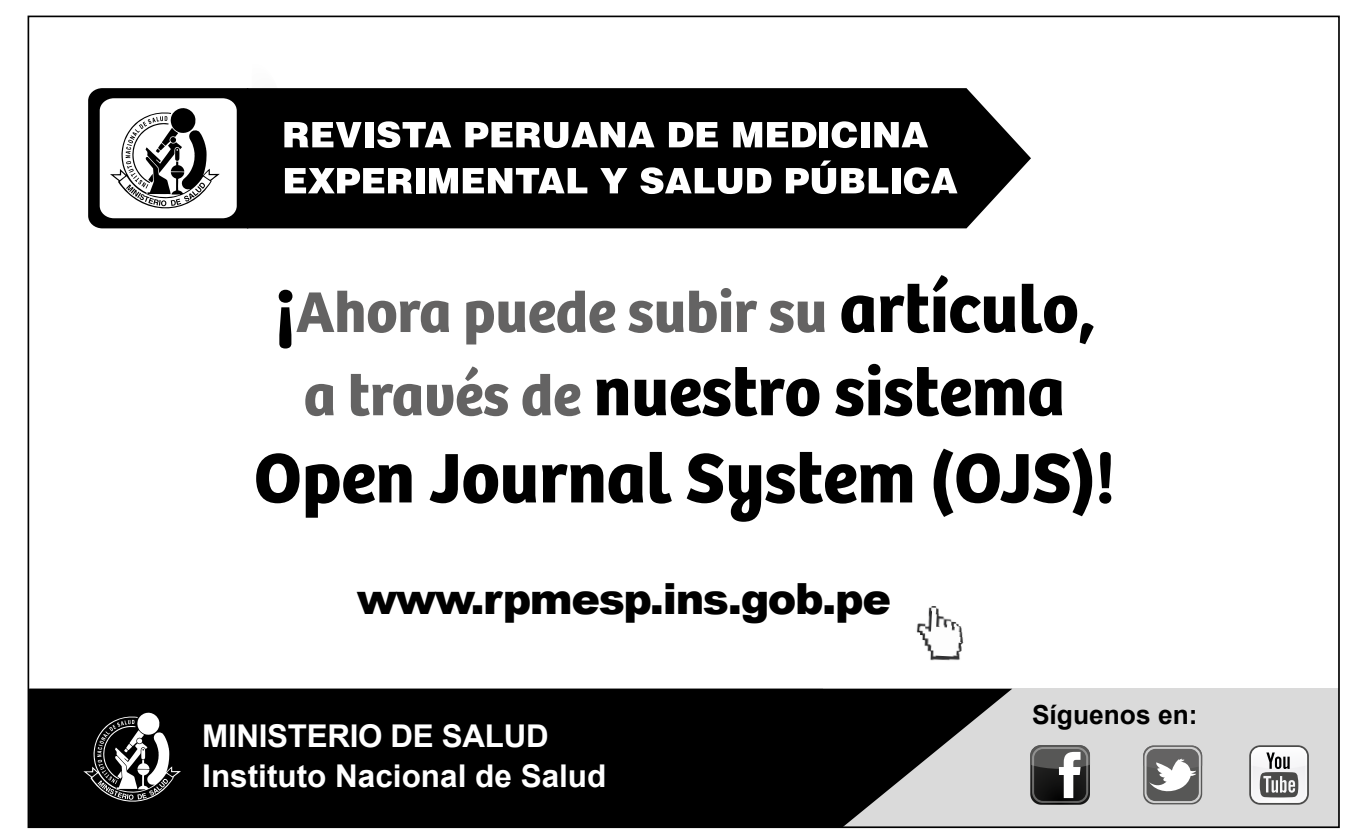

